\title{
Characterization of Extraordinary Transmission for a Single Subwavelength Slit: A Fabry-Pérot-Like Formula Model
}

\author{
Yunping Qi, Jungang Miao, Sheng Hong, Member, IEEE, and Manos M. Tentzeris, Fellow, IEEE
}

\begin{abstract}
Enhanced optical transmission properties of a single subwavelength slit in a metallic film is theoretically studied by the Fabry-Pérot-like formula. By using the analytical model based on a modal expansion method, reflection coefficient of electric field amplitude of surfaces at two slit ends is thoroughly analyzed and an empirical Fabry-Pérot-like formula is derived. Good agreement between our formula and the experimental data is obtained. It is shown that a simple Fabry-Pérot equivalent formula accounts for extraordinary transmission satisfactorily, explaining all of the details of the observed transmission spectra: it is demonstrated that the spacing of two adjacent resonant peaks in the spectra is linearly proportional to the slit width. Also it shows that the linewidth of these resonances increases with increase of the wavelength and is in linear proportion to the slit width. Our Fabry-Pérot-like model can also easily predict on many features of the extraordinary transmission phenomenon.
\end{abstract}

Index Terms-Extraordinary transmission, Fabry-Pérot-like formula, modal expansion method.

\section{INTRODUCTION}

$\mathbf{R}$ ECENTLY, the extraordinary optical transmission (EOT) through narrow subwavelength apertures or slits perforated in a metallic film has attracted a lot of attention [1]-[26]. Since Ebbesen et al. [1] experimentally showed remarkable light transmission through a metallic film with two-dimensional (2-D) array of holes, the analogous structure with 1-D array of subwavelength slits has been analyzed by many researchers [3], [4], [7]-[11], [13], [16], [18], [20], [21], [23], [25], [26]. Many different physical mechanisms of extraordinary optical transmission in slit structures have been proposed. M. M. J. Treacy presented a mechanism based on dynamical diffraction theory [10]. Another explanation is the excitation of surface-plasmon polaritons (SPPs) along the metal-dielectric interfaces [2],

Manuscript received April 15, 2010; revised August 11, 2010; accepted September 11, 2010. Date of publication November 01, 2010; date of current version December 10, 2010. This work was supported by the National Natural Science Foundation of China under Contract 60771012.

Y. Qi is with the School of Electronics and Information Engineering, Beihang University, Beijing 100083, China, and also with the School of Physics and Electronic Engineering, Northwest Normal University, Lanzhou 730070, China.

J. Miao is with the School of Electronics and Information Engineering, Beihang University, Beijing 100083, China.

S. Hong is with the School of Reliability and System Engineering, Beihang University, Beijing 100083, China (e-mail: fengqiao1981@gmail.com).

M. M. Tentzeris is with GEDC/ECE, Georgia Institute of Technology, Atlanta, GA, 30332-250 USA.

Color versions of one or more of the figures in this paper are available online at http://ieeexplore.ieee.org.

Digital Object Identifier 10.1109/TMTT.2010.2083890
[3], [20]-[23]. From a microwave engineering point of view, Medina et al. explained extraordinary transmission through arrays of holes or slits from a simple transmission-line equivalent circuit theory perspective [24], [26].

Schouten et al. [18] presented an experimental and theoretical study of extraordinary optical transmission in a structure of thin metal screen perforated by two subwavelength slits and Zhou $e t$ $a l$. [23] revisited the plasmon-assisted Young's two-slit transmission experiment by employing the finite-difference time-domain (FDTD) algorithm [27]. ${ }^{1}$ When the transmissivity of an isolated slit was adjusted to maximum, they observed total transmissivity is qualified to the contribution from a single slit alone [23]. Therefore, investigations on properties of the light passing through a single metallic slit are essential for understanding its physical origin of the extraordinary transmission phenomena, which has been analyzed and discussed both experimentally [9], [12] and theoretically [5], [25].

The theory that a single slit is theoretically treated as a FabryPérot resonator formed by the slit with two open ends has been reported to describe transmission resonance of the single slit [3], [5], [7]-[9], [11]-[13], [17], [25]. However, a comprehensive study of the reflection coefficient of high-reflection surfaces at the two slit ends is still insufficient. Since standard Fabry-Pérot formula can not be directly applied to modeling the single slit structure [5], [16], the relationship between the single slit and the conventional Fabry-Pérot resonator is still unclear. There is not yet an equivalent Fabry-Pérot-like formula to give interpretations on the transmittance spectrum characteristics of light through the single slit.

In this work, through an analytical method based on a modal expansion of the electromagnetic fields proposed in the previous theoretical studies [5], [14], [15], [17], we derive electrical reflection coefficient of surfaces at two slit ends as a function of the ratio between the wavelength and the slit width, and present the Fabry-Pérot-like formula with modified Fabry-Pérot parameters. Employing this approach, we can explain all of the details of the observed transmission spectra of the single slit and give clear physical interpretations on the spacing of two adjacent resonant transmission peaks and the linewidth of the resonant transmission peaks.

This paper is organized as follows. In Section II we comprehensively study the process of the light passing through the slit and analyze the reflection coefficient of the electric field amplitude $(r)$ of surfaces at two slit ends. Then an equivalent

\footnotetext{
${ }^{1}$ In this paper, a commercially available software developed by Rsoft Design Group http://www.rsoftdesign.com is used for the numerical FDTD simulations.
} 

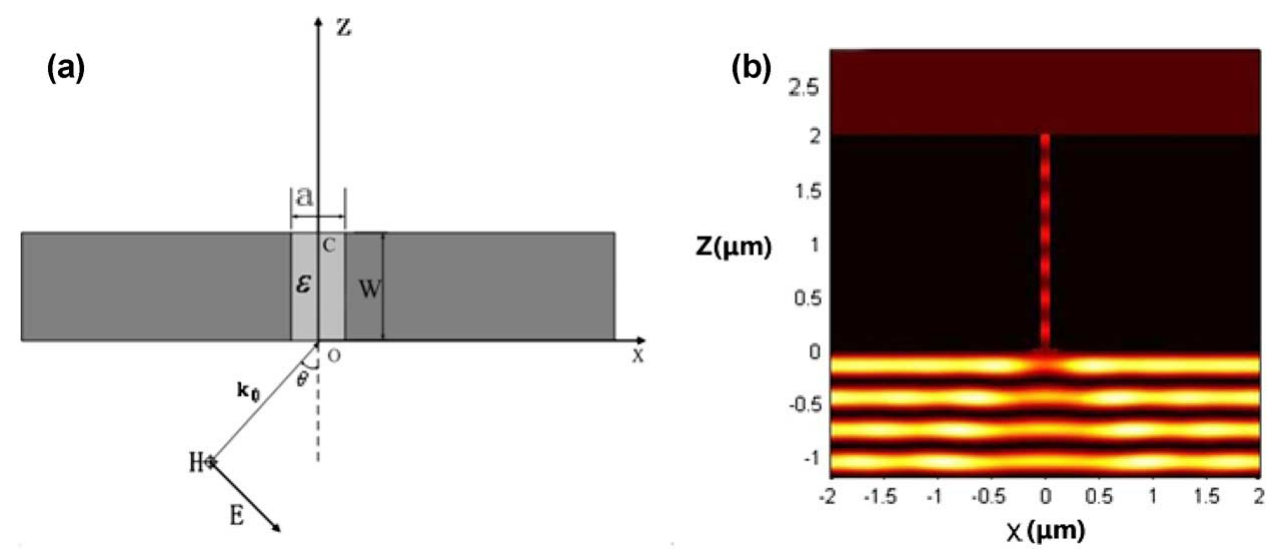

Fig. 1. (a) Schematic diagram of the single subwavelength metal slit in a thick metal screen with the definition of the parameters: the metallic screen thickness, i.e., the slit depth $(W)$, the slit width $(a)$, the incident angle $(\theta)$ and the wave vector $\left(k_{0}\right)$. Dark gray corresponds to perfect electrical conductor, while light gray corresponds to in-slit dielectric characterized by a dielectric constant $\epsilon$. (b) Contour plot of the electric field intensity distribution of a single metallic slit defined by $a=0.2 \mu \mathrm{m}, W=2 \mu \mathrm{m}$. Incident TM-polarized plane wave with $\lambda_{0}=0.6 \mu \mathrm{m}$, having incident angle $\theta=0$ with the $\mathrm{z}$ axis. Brighter areas correspond to higher intensity of the electric field and black areas stand for perfect electrical conductor.

Fabry-Pérot-like theoretical formula describing the normalized transmissivity of a single metal slit is presented. In Section III, this approach is applied to the subwavelength regime subsequently, where the theoretical results are calculated numerically and compared with the results based on a modal expansion [5], [17] and available experimental data [9]. Good agreement between our Fabry-Pérot-like formula and the experimental data is obtained. As a result, it easily explains many features of the resonant transmission phenomenon of a single slit. We have discussed the transmission resonance spectra in the subwavelength regime. It is demonstrated that the spacing of two adjacent resonant peaks is linearly proportional to the slit width. Also it shows that the linewidth of these resonances increases with increase of the wavelength and is in linear proportion to the slit width too. Finally, we give the conclusions in Section IV.

\section{THEORETICAL FORMALISM}

We study a single subwavelength slit perforated in a metallic film, assuming that the metallic film is perfect electrical conductor (PEC). The geometry is sketched in Fig. 1(a), i.e., a Cartesian coordinate system as shown, the coordinate origin locates in the middle axis of the single slit and the point $\mathrm{C}$ is at the exit of the single slit $(x=0, z=W)$. Dark gray corresponds to perfect electrical conductor regions, while light gray corresponds to in-slit dielectric region characterized by a dielectric constant $\epsilon$. The thickness of metallic screen (the slit depth) and slit width are denoted as $W$ and $a$, respectively. Hereby we only consider the TM-polarized (magnetic field $\mathbf{H}$ parallel to $y$ axis) monochromatic plane wave having incident angle $\theta$ with the $z$ axis. The wave vector is represented as $\mathbf{k}_{0}$.

\section{A. Modeling of Extraordinary Transmission of a Single Slit}

First, consider a single slit with the slit width $a=0.2 \mu \mathrm{m}$, the slit depth $W=2 \mu \mathrm{m}$, dielectric material inside the slit is air with dielectric constant $\epsilon=1$, and a TM-polarized plane wave with the wavelength of $\lambda_{0}=0.6 \mu \mathrm{m}$ normally illuminates the slit, we analyze the resonant transmission effect of the slit by virtue of the electromagnetic modal expansion method that was presented in the literature [5], [14], [15], [17]. From the analytical model [17], the $y$ component $H_{y}$ of the magnetic field, the $x$ component $E_{x}$ and $z$ component $E_{z}$ of the electric field inside the slit $(-a / 2 \leq x \leq a / 2$ and $0 \leq z \leq W)$ can be deduced as follows:

$$
\begin{aligned}
H_{y}^{(i n s i d e)}(x, z)= & \sum_{n \geq 0} \frac{1}{\sqrt{\gamma_{n} a}} \cos \left[\frac{n \pi}{a}\left(x+\frac{a}{2}\right)\right] \cdot \frac{i k_{0} \epsilon}{\beta_{n}} \\
\times & {\left[\frac{E_{n}^{I} e^{-i \beta_{n} W}-E_{n}^{O}}{2 \sin \left(\beta_{n} W\right)} e^{i \beta_{n} z}\right.} \\
& \left.+\frac{E_{n}^{I} e^{i \beta_{n} W}-E_{n}^{O}}{2 \sin \left(\beta_{n} W\right)} e^{-i \beta_{n} z}\right] \\
E_{x}^{(\text {inside })}(x, z)= & \sum_{n \geq 0} \frac{1}{\sqrt{\gamma_{n} a}} \cos \left[\frac{n \pi}{a}\left(x+\frac{a}{2}\right)\right] \cdot i \\
\times & {\left[\frac{E_{n}^{I} e^{-i \beta_{n} W}-E_{n}^{O}}{2 \sin \left(\beta_{n} W\right)} e^{i \beta_{n} z}\right.} \\
& \left.-\frac{E_{n}^{I} e^{i \beta_{n} W}-E_{n}^{O}}{2 \sin \left(\beta_{n} W\right)} e^{-i \beta_{n} z}\right] \\
E_{z}^{(i n s i d e)}(x, z)= & \sum_{n \geq 0} \frac{n \pi}{a \beta_{n} \sqrt{\gamma_{n} a}} \sin \left[\frac{n \pi}{a}\left(x+\frac{a}{2}\right)\right] \\
\times & {\left[\frac{E_{n}^{I} e^{-i \beta_{n} W}-E_{n}^{O}}{2 \sin \left(\beta_{n} W\right)} e^{i \beta_{n} z}\right.} \\
& \left.+\frac{E_{n}^{I} e^{i \beta_{n} W}-E_{n}^{O}}{2 \sin \left(\beta_{n} W\right)} e^{-i \beta_{n} z}\right]
\end{aligned}
$$

where the amplitudes $E_{n}^{I}, E_{n}^{O}$ are calculated by the method of moments [28], $\beta_{n}=\sqrt{k_{0}^{2} \epsilon-(n \pi / a)^{2}}$ and $\gamma_{n}$ refers to the normalization factors when solving the Helmholtz equation of $H_{y}(x, z)$ inside the slit and it is equal to $1 / 2$ if $n \neq 0$ and 1 if $n=0$.

In the subwavelength regime with our parameters setting, $\lambda_{0} / a=3>2$, the fundamental mode $(n=0)$ of transmission have dominating contribution to the transmittance for a single slit, other transmission modes are evanescent (surface waves, corresponding to surface plasmons for finite conductivity) [5]. 
So the value of $x$ component of the electric field intensity on the center axis $(x=0)$ of the slit can be written in a simpler form

$$
E_{x}(0, z)=\frac{2}{I_{0} \sin \left(\beta_{0} W\right)}\left\{E_{0}^{I} \sin \left[\beta_{0}(W-z)\right]+E_{0}^{O} \sin \left(\beta_{0} z\right)\right]
$$

and $z$ component is zero everywhere inside the slit. $E_{0}^{I}, E_{0}^{O}$ can be obtained as follows:

$$
E_{0}^{I}=\frac{\left(f_{0}+g_{00}\right) I_{0}}{\left(f_{0}+g_{00}\right)^{2}-g_{0}^{v 2}}
$$

is related to the scattering for the fundamental mode of transmission at the input surface of the slit, while

$$
E_{0}^{O}=-\frac{g_{0}^{v} I_{0}}{\left(f_{0}+g_{00}\right)^{2}-g_{0}^{v 2}}
$$

corresponds to the scattering at the output surface of the slit, where

$$
f_{0}=\frac{i k_{0} \epsilon}{\beta_{0}} \cot \left(\beta_{0} W\right)
$$

is associated with admittance of the fundamental mode, and

$$
g_{0}^{v}=\frac{k_{0} \epsilon}{i \beta_{0} \sin \left(\beta_{0} W\right)}
$$

denotes the bridge variable of the fundamental mode, connecting mode amplitude in the input surface with the output side

$$
g_{00}=\int_{-a / 2}^{a / 2} d x \int_{-a / 2}^{a / 2} d x_{1} G\left(x, x_{1}, k_{0}\right) / a
$$

is the self-interaction term of the fundamental mode coupled inside the slit and

$$
I_{0}=2 \int_{-a / 2}^{a / 2} d x \exp \left[i k_{0} \sin (\theta) x\right] / \sqrt{a}=\frac{4 \sin \left(k_{0} \sin \theta \cdot a / 2\right)}{\sqrt{a} k_{0} \sin \theta}
$$

is the overlap integral between the incident plane wave and the fundamental mode, which reduces to $2 \sqrt{a}$ in the limit of slit width much smaller than the free-space wavelength or normal incidence having incident angle $\theta=0$ with the $\mathrm{z}$ axis. Where $\beta_{n}=\sqrt{k_{0}^{2} \epsilon-(n \pi / a)^{2}}$, so $\beta_{0}=\sqrt{k_{0}^{2} \epsilon}$ and function $G\left(x, x_{1}, k_{0}\right)=(\pi / \lambda) H_{0}^{(1)}\left(k_{0}\left|x-x_{1}\right|\right)$ is the two-dimensional Green's function corresponding to the vacuum.

It is worth noting that the variable $\lambda / a$, the ratio between the wavelength $(\lambda)$ of the incident plane wave and the slit width $(a)$, is very important. The dimensionless variable denoted as $s$ is named as the wavelength-width ratio.

Then we can write $g_{00}$ to an another form

$$
g_{00}=\frac{\pi}{4 s} \int_{-1}^{1} d x \int_{-1}^{1} d x_{1} H_{0}^{(1)}\left[\frac{\pi}{s}\left(x-x_{1}\right)\right]
$$

expedient for calculations. Therefore, the electric field intensity distribution around and inside the single slit is shown in the contour plot of Fig. 1(b). It is apparent from Fig. 1(b) that an open-ended slit cavity supports a series of standing wave modes of the propagating electric field inside the slit region $(0 \leq z \leq$ $W)$, and provides strong resonant enhancement.

\section{B. Reflection Coefficient of the Electric Amplitude at Slit Ends}

As illustrated in Fig. 1(b), it is speculated that there are very thin coating slabs near the entrance (exit) of the slit, in which the incident plane wave passing through the narrow slit is mainly scattered and almost perfectly reflected at both ends. Hereafter we refer the coating slab near the entrance (exit) to the entrance (exit) scattering region. And it is presumed that the actual physical process of the light through a single slit is as follows.

Assuming the amplitude of the electric field intensity of the incident TM-polarized plane wave is $E^{(i)}$, when the plane wave impinges on the slit entrance, it is scattered and divided into two parts in the entrance scattering region. One part goes directly into the slit area with the electric field amplitude $E^{(i)} t^{\prime}$ and the other part is reflected with the electric field $E^{(i)} r^{\prime}$, where $t^{\prime}\left(r^{\prime}\right)$ is the transmission (reflection) coefficient of the electric field of the entrance scattering region, defined as the ratio of the transmission (reflection) electric field amplitude to the incident field amplitude. Propagating wave $E^{(i)} t^{\prime}$ inside the slit continues to go forward with lossless transmission (perfect electrical conductor) and reaches the exit scattering region, and then is scattered by the exit scattering region and divided into two parts too. One goes directly into the outside of slit with the electric field amplitude $E^{(i)} t t^{\prime}$, where $t(r)$ is the transmission (reflection) coefficient of the electric field from the in-slit area to the outside of slit. Similarly, the electric field amplitude of the second outgoing wave of the single slit from the exit scattering region is $E^{(i)} t t^{\prime} r^{2}$.

Compared to the first outgoing wave with the amplitude $E^{(i)} t t^{\prime}$, the second outgoing wave is reflected twice more by the scattering regions and travels a round-trip longer with an optical path length $\Delta=2 n_{\text {inside }} W$ inside the slit, taking the perpendicular incident into account, where $n_{\text {inside }}=\sqrt{\epsilon}$ is the effective refractive index of the dielectric inside the slit. Moreover the phase difference caused by the optical path difference is

$$
\delta=k_{0} \Delta=2 k_{0} n_{\text {inside }} W=\left(4 \pi / \lambda_{0}\right) n_{\text {inside }} W .
$$

As a result, the complex amplitude of the electric field of the second outgoing transmission wave is

$$
\mathbf{E}_{2}^{(t)}=r^{2} t t^{\prime} E^{(i)} e^{-i \delta} .
$$

Then, we obtain the transmissivity of the metallic single slit $(T)$ as written in the conventional Fabry-Pérot formula

$$
T(s)=\frac{T_{0}(s)}{1+F(s) \cdot \sin ^{2}(\delta / 2)}
$$

where $F(s)=4 r^{2}(s) /\left[1-r^{2}(s)\right]^{2}$ is named as hyperfine coefficient, $r(s)$ is the reflection coefficient of electric field am- 
(a)

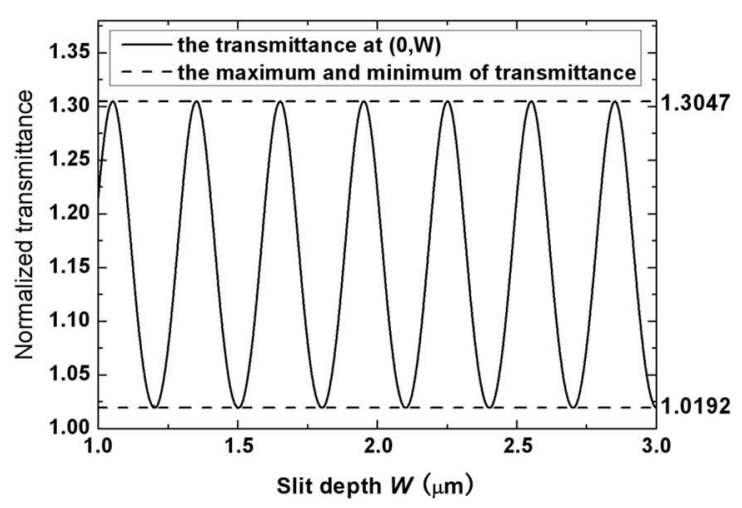

(b)

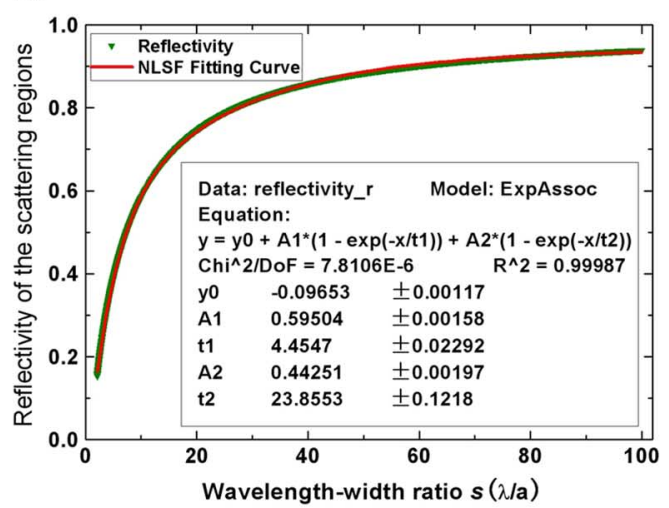

Fig. 2. (a) Normalized transmittance $(T)$ at the central exit $C(0, W)$ of the single slit as a function of the slit depth (W) for the given parameters: the slit width $a=0.2 \mu \mathrm{m}$, the dielectric material inside the slit is air $(\epsilon=1)$, normal incident TM polarization plane wave with $\lambda_{0}=0.6 \mu \mathrm{m}$. (b) Reflection coefficient $(r)$ of electric field amplitude of the scattering regions as a function of the wavelength-width ratio $(s)$. The triangle symbols represent the numerical calculation data from Eq. (18) and the solid line is the nonlinear least square fitting curve. Normalized transmittance, reflectivity of the scattering regions and wavelength-width ratio have dimensionless unit.

plitude from the inside of slit to the outside of slit and function $T_{0}(s) \propto E^{(i)} E^{(i) *}$ is the amplitude of the resonances associated with the incident electric field intensity value. It is crucial that $T_{0}(s)$ and $r(s)$ are not constant but as functions of the wavelength-width $s$.

Seen from (14), when $\sin \left(k_{0} n_{\text {inside }} W\right)=0$, the transmissivity of the single slit $(T)$ can reach its maximum $T_{\max }(s)=T_{0}(s)$. Therefore, the resonant wavelength of the $m$ th order mode $\left(\lambda_{m}\right)$ of the single slit are given by the following equation:

$$
\lambda_{m}=\frac{2 n_{\text {inside }} W}{m}, \quad(m=0,1,2,3, \ldots)
$$

where $m$ is the order mode number, $n_{\text {inside }}=\sqrt{\epsilon}$ is the refractive index. And when $\sin \left(k_{0} n_{\text {inside }} W\right)=1$, the transmissivity of the single slit $(T)$ can reach its minimum

$$
T_{\text {min }}(s)=\frac{1}{1+F(s)} T_{0}(s)=\left[\frac{1-r^{2}(s)}{1+r^{2}(s)}\right]^{2} T_{0}(s)
$$

so, the ratio of the transmissivity maximum to minimum of the single slit is

$$
H(s)=\frac{T_{\max }(s)}{T_{\min }(s)}=\left[\frac{1+r^{2}(s)}{1-r^{2}(s)}\right]^{2}
$$

and the positive reflection coefficient of the electric field amplitude of the scattering regions $(r)$ can be calculated

$$
r(s)=\sqrt{\frac{\sqrt{H(s)}-1}{\sqrt{H(s)}+1}} .
$$

We focus on the $x$ component $\left(E_{x}\right)$ of the electric field intensity $E_{x}(0, W)=E_{0}^{O} \phi_{0}(0)$ at the point (C) at the central exit of the single slit $(x=0, z=W)$, where $\phi_{0}(0)=2 / I_{0}$ and $I_{0}=2 \int_{-a / 2}^{a / 2} d x(1 / \sqrt{a}) \exp \left[i k_{0} \sin (\theta x)\right]=2 \sqrt{a}$. So the transmissivity at the point $\mathrm{C}(0, W)$ is

$$
T=E_{0}^{O} E_{0}^{O *} \phi_{0}(0) \phi_{0}^{*}(0) .
$$

Fig. 2(a) graphs the transmittance $(T)$ at the point $\mathrm{C}(0, W)$ as a function of the slit depth $(W)$ for the given parameters unchange in Fig. 1(b) except for the variable slit depth $(W)$. For instance, as shown in Fig. 2(a), $s=\lambda / a=3$, the maximum of the transmittance is the $T_{\max }(3)=1.3047$ and the minimum is $T_{\min }(3)=1.0192$, so $H(3)=T_{\max }(3) / T_{\min }(3) \approx 1.28$, the reflection coefficient of electric field of the scattering region is calculated to be $r(3)=\sqrt{(\sqrt{H(3)}-1) /(\sqrt{H(3)}+1)}=$ $24.83 \%$.

In the same way, by utilizing the above formulae to calculate ratio of the maximum to the minimum of the transmittance $H(s)$ at the point $\mathrm{C}(0, W)$ with different wavelengths of incident radiation and slit widths, we explore the reflection coefficient of the scattering region at the open ends of the slit as a function of the wavelength-width ratio $(s)$, as shown in Fig. 2(b). The triangle symbols represent the numerical calculation data deriving from (18) and the reflectivity increases damped exponentially with the wavelength-width ratio $(s)$. Correspondingly, we use the exponential associate function $r(s)=$ $y_{0}+A_{1}\left(1-e^{-s / t_{1}}\right)+A_{2}\left(1-e^{-s / t_{2}}\right)$ to fit the numerical calculation results, the parameters using the nonlinear least square fitting (NLSF) algorithm are obtained as follows:

$$
\begin{aligned}
y_{0} & =-0.09653 \pm 0.00117 \\
A_{1} & =0.59504 \pm 0.00158 \\
A_{2} & =0.44251 \pm 0.00197 \\
t_{1} & =4.4547 \pm 0.02292 \\
t_{2} & =23.8553 \pm 0.1218
\end{aligned}
$$

with the level of $99.99 \%$ confidence. As you can see from Fig. 2(b), the solid line is the NLSF curve. So the empirical equation of the reflection coefficient of the scattering regions in the subwavelength regime is

$r(s)=0.941-0.595 \times e^{-s / 4.45}-0.443 \times e^{-s / 23.86}, \quad(s>2)$.

In [25], the electromagnetic wave with the wavelength $\lambda_{0}=$ $0.8 \mu \mathrm{m}$ illuminates a slit perforated in silver film with $a=$ 
$0.1 \mu \mathrm{m}, W=2 \mu \mathrm{m}$, i.e., the wavelength-width ratio $s=8$. The reflectivity is estimated by the FDTD algorithm [27] to be $r \approx 0.575$, while according to our theory the reflectivity $r(8)=$ 0.527 calculated from (20). They are almost same but the difference is mainly contributed by the finite conductivity effects such as skin depth considering silver film in [25]. If the dimensions of the single slit are several times larger than the skin depth of the slit, the approximate model discussed in this paper provides quantitative results for metals with finite conductivity, such as silver or aluminum. This reflectivity $r$ value is very large, which implies that the two scattering regions have abnormal high reflection coefficient of electric field amplitude in the subwavelength regime through strong diffraction on resonance.

It is concluded that the conjecture from beginning that the single slit can be treated as a Fabry-Pérot resonator consisting of a dielectric body sandwiched by thin high-reflectivity coating slabs on its two surfaces, is verified to be appropriate. And the reflection coefficient of the coating is not yet constant, which is different from the conventional Fabry-Pérot interferometer, increasing with the increase of wavelength-width ratio $(s)$.

\section{Modified Fabry-Pérot Equivalent Formula}

When the ratio $a / \lambda$ approaches to zero, i.e., $a / \lambda \rightarrow 0$, resonant transmission peak locations of the single slit are in good match with the resonant wavelengths of the simple Fabry-Pérot model shown by (15). While $a / \lambda$ is small but not zero, the transmission peaks are shifted and broadened. For a single slit in a perfect electrical conductor the degree of systematic shift in wavelength-width ratio towards longer wavelengths is estimated by Takakura [5] to be

$$
\frac{s_{\text {shift }}}{s}=\frac{2(a / W)[\ln (\pi / s)-3 / 2]}{2(a / W)[\ln (\pi / s)-1 / 2]-\pi}
$$

where $a$ is the slit width, $W$ is the slit depth, and $s$ is the wavelength-width ratio.

Another difference from the conventional Fabry-Pérot formula is that the normalized transmittance value of resonance peaks amplitude $T_{0}(s)$ in (14) is linearly proportional to the wavelength-width ratio in our modified Fabry-Pérot formula as shown in Fig. 3. Employing the electromagnetic modal expansion method, we calculate the amplitude of the resonant transmission peaks demonstrated by the triangle symbols as depicted in Fig. 3. Then we use linear fitting on the amplitude of resonant transmission peaks and obtain the linear regression function of $T_{0}(s)$ with the variable wavelength-width ratio $(s)$

$$
T_{0}(s)=0.0096+0.3183 \times s .
$$

As shown in Fig. 3, the solid lines are the linear fitting curves of the resonances. From Fig. 3(a) where $W=28.2 \mathrm{~mm}, a=$ $75 \mu \mathrm{m}$ and in the Fig. 3(b) where $W=2.0 \mu \mathrm{m}, a=8.0 \mathrm{~nm}$, the same range of wavelength-width ratio from 55 to 260 in the subwavelength regime, we also note that the linear regression coefficient $(A \approx 0.0096)$ and $(B \approx 0.3183)$ of $T_{0}(s)=$ $A+B \times s$ are independent of the slit width $(a)$ and the slit depth $(W)$ concluded from results of linear regression fitting with different slit widths and slit depths. We now reduce (14) to a modified Fabry-Perot formula of the normalized transmittance of the

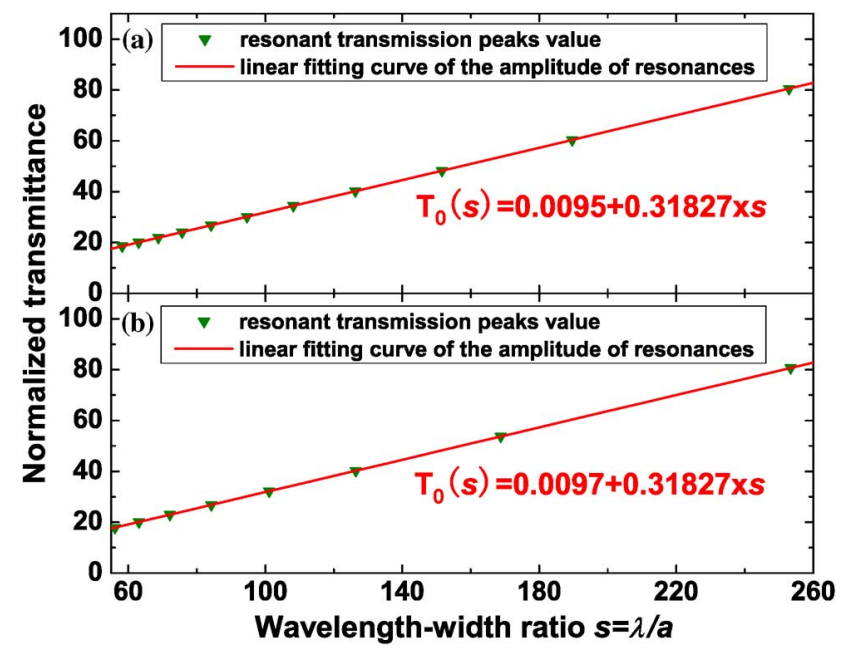

Fig. 3. Normalized transmittance of resonance peaks of the single slit as a linear function of the wavelength-width ratio $(s)$. The triangle symbols represent amplitude of the resonant transmission peaks while the solid lines are the linear fitting curves of the peaks computed with (a) $W=28.2 \mathrm{~mm}, a=75 \mu \mathrm{m}$ and (b) $W=2.0 \mu \mathrm{m}, a=8.0 \mathrm{~nm}$, refractive index of dielectric inside the slit $n_{\text {inside }}=1$ and normal incidence is assumed. Normalized transmittance and wavelength-width ratio have dimensionless unit.

metallic single slit $(T)$ as a function of the wavelength-width ratio $(s)$

$$
T(s)=\frac{0.0096+0.3183 \times s}{1+F(s) \cdot \sin ^{2}\left[\frac{2 \pi \cdot n_{\text {inside }} W}{\left(s-s_{\text {shift }}\right) a}\right]}
$$

where $F(s)=4 r^{2}(s) /\left[1-r^{2}(s)\right]^{2}, n_{\text {inside }}$ is the refractive index of dielectric inside the slit, $r(s)$ is derived from the empirical equation (20), $a$ is the slit width, $W$ is the slit depth, and $s_{\text {shift }}$ as the result of an aperture effect is calculated from the (21).

\section{NUMERICAL RESULTS AND DISCUSSION}

In order to compare with experimental data published by Yang and Sambles [9] and numerical results of electromagnetic modal expansion method published by J. Bravo-Abad et al. [17], we adopt the same metal single slit parameters by assuming $a=75 \mu \mathrm{m}, W=28.2 \mathrm{~mm}$ and the same range of wavelengths in the subwavelength regime.

First we compare two theoretical results of our Fabry-Pérotlike formula and electromagnetic modal expansion method [17]. From the (23), we could get the numerical result of our FabryPérot-like formula about the normalized transmittance $(T)$ of the single slit as a function of the wavelength-width ratio $(s)$, as illustrated with the dash-dotted line in Fig. 4, while the solid line is result of the electromagnetic modal expansion redrawn from [17]. For observing the difference in more detail on the resonant transmission peaks of two theoretical methods, inbox of Fig. 4 shows the transmission peak at $s=253$. Consequently, we can see that the results of two theoretical methods are always in agreement and residual mismatch is caused by no taking into account the higher orders in the perturbation calculation in (21) and the other (evanescent) cavity modes [5].

Then we compare result of our Fabry-Pérot-like formula with experimental data adopted from Yang and Sambles [9] and sim- 


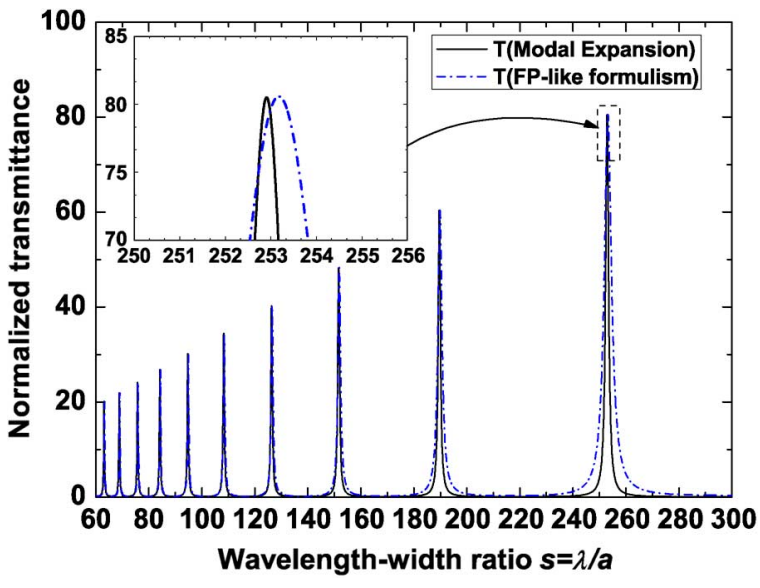

Fig. 4. Main: comparison of the normalized transmittance $(T)$ of the single slit calculated from the Fabry-Pérot-like formula and the electromagnetic modal expansion. The numerical result of our Fabry-Pérot-like theory formula plotted from the Eq. (23) (dash lines) is in agreement with analytical results of the electromagnetic modal expansion [17] (solid line), computed with $W=28.2 \mathrm{~mm}$ and $a=75 \mu \mathrm{m}$. Inbox: comparison of the resonant transmission peak at $s=$ 253 with wavelength-width ratio as abscissas. Normalized transmittance and wavelength-width ratio have dimensionless unit.

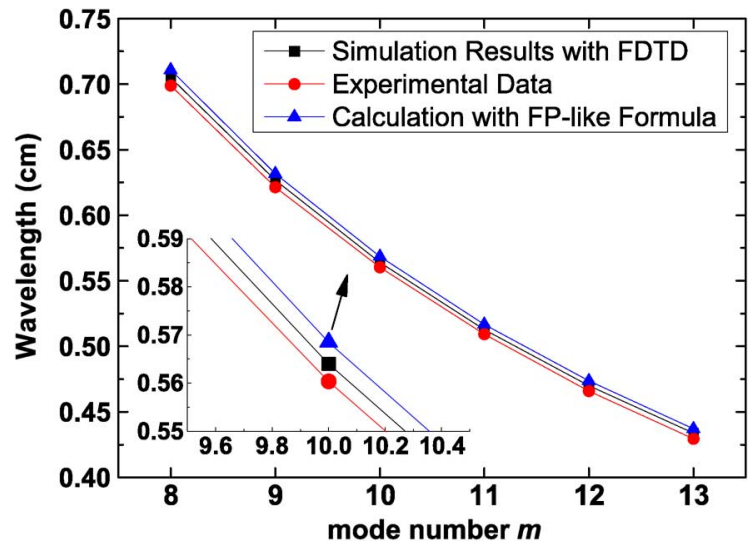

Fig. 5. Main: comparison of our Fabry-Pérot-like theoretical results with experimental data of resonant wavelengths and simulation results with FDTD, with the order mode number as abscissa, computed with $W=28.2 \mathrm{~mm}$, $a=75 \mu \mathrm{m}$. Inbox: Fabry-Pérot resonance wavelength details of the 10 'th resonance mode number.

ulation results employing the FDTD algorithm [27]. As shown in Fig. 5, the comparison of our Fabry-Pérot-like theoretical results with the experimental data and simulation results is plotted with resonant mode numbers as abscissa, from 8 to 13 referred to (15). Inbox of Fig. 5 shows the details of the Fabry-Pérot resonance wavelength with 10'th order mode number. The six filled circles are the experimental data of resonance wavelengths adopted from Yang and Sambles [9], the six filled triangles on the upper are the values of our Fabry-Pérot-like formula calculated from (23) and the six filled squares are the simulation results with FDTD [27].

As can be seen in this figure, this tiny mismatch is associated with the finite conductivity of the aluminum used in experiment [9]. It is apparent from Fig. 5 that the relative deviation of the resonance wavelengths of our Fabry-Pérot-like formula to the experimental data less than $2.0 \%$ is acceptable.

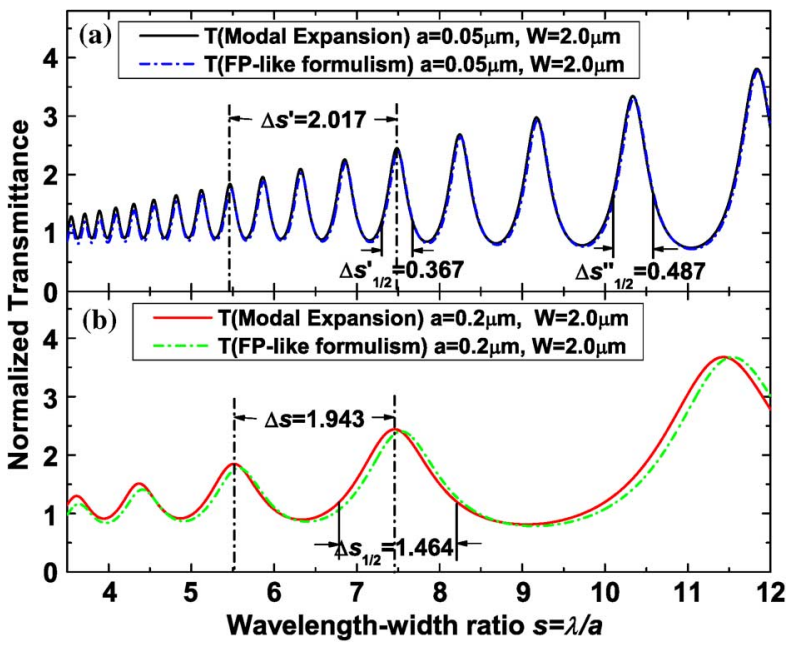

Fig. 6. Normalized transmittance of a single slit as a function of the wavelength-width ratio of the incident radiation, computed with the same range from 3.5 to 12 with wavelength-width ratio $(s)$ as abscissas, the same slit depth $W=2 \mu \mathrm{m}$ and two different values of the slit width $a, 0.05 \mu \mathrm{m}$ (a) and 0.2 $\mu \mathrm{m}$ (b). Normal incidence and $\epsilon=1$ inside the slit is assumed. Normalized transmittance and wavelength-width ratio have dimensionless unit.

So we conclude that the extraordinary optical transmission of light through a single subwavelength slit can be depicted by the Fabry-Pérot-like formula model. And the transmission characteristics (linewidth of resonances, spacing of two adjacent resonant peaks, etc) of the resonant transmission peaks can also be calculated and described analytically by the equivalent FabryPérot formula.

\section{A. Spacing of Two Adjacent Resonant Transmission Peaks}

Now, we again focus on the single slit with $W=2 \mu \mathrm{m}$ and $a=0.2 \mu \mathrm{m}$ as discussed in Section II. For comparing, we consider another single slit with same slit depth $W=2 \mu \mathrm{m}$ and different slit width $a=0.05 \mu \mathrm{m}$. There is same dielectric material air inside the slit with dielectric constant $\epsilon=1$. As shown in Fig. 6(a) with the slit width $a=0.05 \mu \mathrm{m}$ and Fig. 6(b) with the slit width $a=0.2 \mu \mathrm{m}$, computed with the same range from 3.5 to 12 with wavelength-width ratio $(s)$ as abscissas, the black (a) and red (b) continuous lines are analytical results of the electromagnetic modal expansion method and the blue (a) and green (b) dash-dotted lines are the numerical results of Fabry-Pérot-like formula plotted from the (23).

Supposing that two adjacent resonant wavelengths are $\lambda$ and $\lambda^{\prime}=\lambda+\Delta \lambda$, because all the slit resonance peaks are shifted towards longer wavelengths, so $\lambda_{F P}=\lambda-\lambda_{\text {shift }}$ and $\lambda_{F P}^{\prime}=$ $\lambda-\lambda_{\text {shift }}+\Delta \lambda$ are solutions of (15), where the mode number $m$ and $m^{\prime}=m-1$ correspond to Fabry-Pérot resonant wavelength $\lambda_{F P}$ and $\lambda_{F P}^{\prime}$ respectively.

Then we have the following equation

$$
m \lambda_{F P}=(m-1)\left(\lambda_{F P}+\Delta \lambda\right)
$$

since $m \gg 1$, so

$$
\Delta \lambda=\frac{\lambda_{F P}}{m}=\frac{\lambda_{F P}^{2}}{2 n_{\text {inside }} W}=\frac{\left(\lambda-\lambda_{\text {shift }}\right)^{2}}{2 n_{\text {inside }} W}
$$


the equation for the wavelength-width ratio can be expressed as

$$
\Delta s=\frac{\left(s-s_{\text {shift }}\right)^{2} a}{2 n_{\text {inside }} W}
$$

where $\Delta s$ is the spacing of the adjacent resonant transmission peaks of a single slit and $s_{\text {shift }}$ is calculated from (21). As can be seen in Fig. 6(a) and Fig. 6(b), with the increase of the wavelength-width ratio, the spacing of adjacent resonant transmission peaks increases quadratically.

And (26) shows that supposing that the wavelength-width ratio is same or approximately same, then the interval $(\Delta s)$ is linearly proportional to the slit width $(a)$. For instance, as the Fig. 6(b) $(a=0.2 \mu \mathrm{m})$ shows, the difference of the neighboring resonant transmission peaks at $s_{1}=5.515$ and $s_{2}=7.458$ is $\Delta s=1.943$. Within this range, considering the slit width $a=0.05 \mu \mathrm{m}$ as shown in Fig. 6(a), there are five resonant transmission peaks, that is $s_{1}^{\prime}=5.467, s_{2}^{\prime}=5.863, s_{3}^{\prime}=6.319$, $s_{4}^{\prime}=6.853$ and $s_{5}^{\prime}=7.484$ respectively, whose average interval from each other is $\overline{\Delta s}=\left(s_{5}^{\prime}-s_{1}^{\prime}\right) / 4=2.017 / 4 \approx 0.504$. As a matter of fact, $\Delta s$ of the single slit with $a=0.2 \mu \mathrm{m}$ is about four times as much as that of the single slit with $a=0.05 \mu \mathrm{m}$, which is accordance with the ratio of two slit widths, that is $\Delta s \propto a$. So we can conclude that the spacing of two adjacent resonant peaks in the spectra is obviously linearly proportional to the slit width, with constant slit depth.

\section{B. Full Width at Half Maximum}

It is well known that the linewidth of resonances is quantitatively measured by the full width at half maximum (FWHM) of resonant transmission peaks. It is worth noting that the shift towards longer wavelengths of the resonance peaks is systematic, so the resonances FWHM of conventional Fabry-Pérot theory and modified Fabry-Pérot-like formula have same values. Let $\varepsilon$ stands for the phase FWHM of the resonant transmission peaks and when the phase $\delta=2 m \pi \pm(\varepsilon / 2)$, the normalized transmittance decrease from the peak value to the half value of the maximum, i.e., $T=T_{0}(s) / 2$. Thus, the equation is easy to be obtained from the (14)

$$
\frac{1}{1+F \cdot \sin ^{2}\left[\frac{1}{2}(2 m \pi \pm \varepsilon / 2)\right]}=\frac{1}{2}
$$

accordingly we can obtain the phase FWHM

$$
\varepsilon=4 \arcsin (\sqrt{1 / F}) .
$$

As we know, the phase is in inverse proportion to the wavelength concluded from the equation

$$
\delta=2 k_{0} n_{\text {inside }} W=\frac{4 \pi}{\lambda} \cdot n_{\text {inside }} W
$$

so we can obtain the phase FWHM is

$$
\varepsilon=\frac{4 \pi \cdot n_{\text {inside }} W \cdot \Delta \lambda_{\frac{1}{2}}}{\lambda^{2}}
$$

substituting (28) into the above equation, the corresponding wavelength FWHM can be reduced as

$$
\Delta \lambda_{\frac{1}{2}}=\frac{\lambda^{2}}{\pi n_{\text {inside }} W} \arcsin (\sqrt{1 / F})
$$

and the equation of wavelength-width ratio FWHM can be written as

$$
\Delta s_{\frac{1}{2}}=\frac{s^{2} a}{\pi n_{\text {inside }} W} \arcsin (\sqrt{1 / F})
$$

where $F(s)=4 r^{2}(s) /\left[1-r^{2}(s)\right]^{2}, r(s)$ is calculated by (20).

This is the physical explanation on why the wavelength-width ratio FWHM $\left(\Delta s_{1 / 2}\right)$ increases with the increase of the wavelength-width ratio $(s)$ shown in Fig. 6. For instance, Fig. 6(a) illustrates, computed with the slit width $a=0.05 \mu \mathrm{m}$ and the slit depth $W=2 \mu \mathrm{m}$, the wavelength-width ratio $\operatorname{FWHM}\left(\Delta s_{1 / 2}\right)$ of the two resonant transmission peaks located at $s_{1}=7.484$ and $s_{2}=10.336$ are obtained by substituting $s$ into (32) to be 0.367 and 0.487 , respectively. In addition, we can also see from (32) that as for the same or approximately same wavelength-width ratio, $\Delta s_{1 / 2}$ is in linear proportion to the slit width (a), which is illustrated in Fig. 6. It indicates that the linewidth of the resonance peaks is linearly proportional to the slit width. So in physical application such as high-sensitivity microfluidics detector, the linewidth of the resonance peaks can be adjusted to decrease for higher resolution by reducing the width of the slit.

\section{More Discussion}

It can be seen from (20) that the reflection coefficient $r(s)$ of the electric field amplitude of surfaces at two slit ends can not reach $100 \%$. From the (20), we can conclude that the maximum limit is 0.941 if $s \rightarrow \infty$. It indicates that the high electric field reflective effect of two thin high-reflectivity surfaces of the single slit can not been perfectly achieved. With the increasing of the wavelength-width ratio, the diffraction of two high-reflectivity surfaces is stronger but with a limitation.

In this work, in order to analyze the structure more directly and neglect typical finite conductivity effects such as skin depth, we assume infinite conductivity for the metallic regions. If the dimensions of the single slit are several times larger than the skin depth, the Fabry-Pérot-like formula model proposed in this paper provides at least a good approximate value for the metallic slit with finite conductivity [8], such as silver or aluminum. The role that metal loss plays increasing the reflectivity of electric field amplitude of surfaces at two slit ends. By decreasing the dimensions of the single slit and increasing the skin depth, the metal loss starts having a non-negligible contribution to the reflectivity.

And the limitations of the theory we propose is only applicable in the subwavelength regime, i.e., $\lambda_{0} / a>2$, more complex transmission spectra associated with rapid oscillations appear when the wavelength of light is comparable to the slit width [17].

\section{CONCLUSION}

In this paper, a Fabry-Pérot-like formula model for studying the enhanced optical transmission properties of a subwavelength single slit has been presented. Employing the electromagnetic modal expansion method, we have analyzed the electric field reflectivity of surfaces at two slit ends as a function of the ratio between the wavelength and the slit width and we derived a mod- 
ified Fabry-Pérot-like formula. Good agreement between our Fabry-Pérot-like formula and the experimental data is obtained.

In addition, using the equivalent Fabry-Pérot-like formula is reasonable and particularly expedient for elucidating the transmittance characteristics of the single slit. We have discussed and studied the transmission resonances spectra in the subwavelength regime. It is demonstrated that the spacing of two adjacent resonant peaks is linearly proportional to the slit width. Also it shows that the linewidth of these resonances increases with increase of the wavelength and is in linear proportion to the slit width too. We believe that the proposed theoretical FabryPérot-like formula can deepen the understanding of the intuitionistic physical picture of resonant enhancement transmission of a single slit.

\section{ACKNOWLEDGMENT}

The authors thank Prof. H. Wang from the Department of Physics of Tsinghua University, Beijing, China, and Prof. Yunsong Zhou for their discussions to this research.

\section{REFERENCES}

[1] T. W. Ebbesen, H. J. Lezec, H. F. Ghaemi, T. Thio, and P. A. Wolff, "Extraordinary opticl transmission through sub-wavelength hole arrays," Nature, vol. 391, no. 12, pp. 667-669, Feb. 1998.

[2] H. F. Ghaemi, T. Thio, D. E. Grupp, T. W. Ebbesen, and H. J. Lezec, "Surface plasmons enhance optical transmission through subwavelength holes," Phys. Rev. B, vol. 58, no. 11, pp. 6779-6782, Sep. 1998.

[3] J. A. Porto, F. J. García-Vidal, and J. B. Pendry, "Transmission resonances on metallic gratings with very narrow slits," Phys. Rev. Lett., vol. 83, no. 14, pp. 2845-2848, Oct. 1999.

[4] H. E. Went, A. P. Hibbins, J. R. Sambels, C. R. Lawrence, and A. P. Crick, "Selective transmission through very deep zero-order metallic gratings at microwave frequencies," Appl. Phys. Lett., vol. 77, no. 18, pp. 2789-2791, Oct. 2000.

[5] Y. Takakura, "Optical resonance in a narrow slit in a thick metallic screen," Phys. Rev. Lett., vol. 86, no. 24, pp. 5601-5603, Jun. 2001.

[6] L. Martín-Moreno, F. J. García-Vidal, H. J. Lezec, K. M. Pellerin, T. Thio, J. B. Pendry, and T. W. Ebbesen, "Theory of extraordinary optical transmission through subwavelength hole arrays," Phys. Rev. Lett., vol. 86, no. 6, pp. 1114-1117, Feb. 2001

[7] Q. Cao and P. Lalanne, "Negative role of surface plasmons in the transmission of metallic gratings with very narrow slits," Phys. Rev. Lett., vol. 88, no. 5, pp. 057403-1-057403-4, Feb. 2002.

[8] F. J. García-Vidal and L. Martín-Moreno, "Transmission and focusing of light in one-dimensional periodically nanostructured metals," Phys. Rev. B., vol. 66, pp. 155412-1-057403-10, Oct. 2002.

[9] F. Yang and J. R. Sambles, "Resonant transmission of microwaves through a narrow metallic slit," Phys. Rev. Lett., vol. 89, no. 6, pp. 063901-1-063901-3, Aug. 2002.

[10] M. M. J. Treacy, "Dynamical diffraction explanation of the anomalous transmission of light through metallic gratings," Phys. Rev. B., vol. 66, pp. 195105-1-195105-11, Nov. 2002.

[11] A. Barbara, P. Quémerais, E. Bustarret, and T. Lopez-Rios, "Optical transmission through subwavelength metallic gratings," Phys. Rev. B., vol. 66, pp. 161403-1-161403-4, Oct. 2002.

[12] A. P. Hibbins, J. R. Sambles, and C. R. Lawrence, "Gratingless enhanced microwave transmission through a subwavelength aperture in a thick metal plate," Appl. Phys. Lett., vol. 81, no. 24, pp. 4661-4663, 2002.

[13] A. Barbara, P. Quémerais, E. Bustarrel, T. Lopez-Rios, and T. Fournier, "Electromagnetic resonances of sub-wavelength rectangular metallic gratings," Eur. Phys. J. D, vol. 23, no. 1, pp. 143-154, Sep. 2003.

[14] L. Martín-Moreno, F. J. García-Vidal, H. J. Lezec, A. Degiron, and T. W. Ebbesen, "Theory of highly directional emission from a single subwavelength aperture surrounded by surface corrugations," Phys. Rev. Lett., vol. 90, no. 16, pp. 167401-1-167401-4, Apr. 2003.
[15] F. J. García-Vidal, H. J. Lezec, T. W. Ebbesen, and L. Martín-Moreno, "Multiple paths to enhance optical transmission through a single subwavelength slit," Phys. Rev. Lett., vol. 90, no. 21, pp. 213901-1-213901-4, May 2003.

[16] J. R. Suckling, A. P. Hibbins, M. J. Lockyear, T. W. Preist, J. R. Sambles, and C. R. Lawrence, "Finite conductance governs the resonance transmission of thin metal slits at microwave frequencies," Phys. Rev. Lett., vol. 92, no. 14, pp. 147401-1-147401-4, Apr. 2004.

[17] J. Bravo-Abad, L. Martín-Moreno, and F. J. García-Vidal, "Transmission properies of a single metallic slit: From the subwavelength regime to the geometrical-optics limit," Phys. Rev. E., vol. 69, pp. 0266011-026601-6, Feb. 2004.

[18] H. F. Schouten, N. Kuzmin, G. Dubois, T. D. Visser, G. Gbur, P. F. Alkemade, H. Blok, G. W. 't Hooft, D. Lenstra, and E. R. Eliel, "Plasmon-assisted two-slit transmission: Young's experiment revisited," Phys. Rev. Lett., vol. 94, pp. 053901-1-053901-4, Feb. 2005.

[19] F. J. García-Vidal, E. Moreno, J. A. Porto, and L. Martín-Moreno, "Transmission of light through a single rectangular hole," Phys. Rev. Lett., vol. 95, pp. 103901-1-103901-4, Sep. 2005.

[20] A. P. Hibbins, M. J. Lockyear, and J. R. Sambles, "The resonant electromagnetic fields of an array of metallic slits acting as Fabry-Perot cavities," J. Appl. Phys., vol. 99, pp. 124903-1-124903-5, Jun. 2006.

[21] P. Lalanne, J. P. Hugonin, and J. C. Rodier, "Approximate model for surface-plasmon generation at slit apertures," J. Opt. Soc. Amer. A, vol. 23, pp. 1608-1615, 2006.

[22] A. Y. Nikitin, F. Lopez-Tejeira, and L. Martín-Moreno, "Scattering coefficients of surface plasmon polaritons impinging at oblique incidence onto one-dimensional surface relief defects," Phys. Rev. B., vol. 75, pp. 081405(R)-1-081405(R)-4, Feb. 2007.

[23] Y.-S. Zhou, B.-Y. Gu, S. Lan, and L.-M. Zhao, "Time-domain analysis of mechanism of plasmon-assisted extraordinary optical transmission," Phys. Rev. B., vol. 78, pp. 081404(R)-1-081404(R)-4, Aug. 2008.

[24] F. Medina, F. Mesa, and R. Marqués, "Extraordinary transmission through arrays of electrically small holes from a circuit theory perspective," IEEE Trans. Microw. Theory Tech., vol. 56, no. 12, pp. 3108-3120, Dec. 2008.

[25] Y.-S. Zhou, B.-Y. Gu, H.-Y. Wang, and S. Lan, "Multi-reflection process of extraordinary optical transmission in a single subwavelength metal slit," Euro. Phys. Lett., vol. 85, pp. 24005-p1-24005-p5, Jan. 2009.

[26] F. Medina, F. Mesa, and D. C. Skigin, "Extraordinary transmission through arrays of slits: A circuit theory model," IEEE Trans. Microw. Theory Tech., vol. 58, no. 1, pp. 105-115, Jan. 2010.

[27] K. S. Yee, "Numerical solution of initial boundary value problems involving Maxwell's equations in isotropic media," IEEE Trans. Antennas Propag., vol. 14, no. 3, pp. 302-307, May 1966.

[28] R. F. Harrington, Field Computation by Moment Methods. New York: MacMillan, 1968.

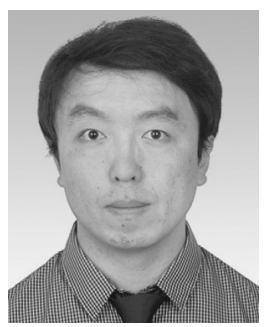

Yunping Qi was born in Gansu, China, in May 1981. He received the B.S. degree in physics from the Northwest Normal University, Lanzhou, China in 2003, and the M.S. degree in communication and information system from the Beihang University, Beijing, China, in 2006. In 2008, He was a Ph.D. candidate in Computational Electromagnetics and Microwave Remote Sensing from the Beihang University, Beijing, China.

In 2006, he began teaching with the Northwest Normal University, where he is currently a Lector. In 2008, He became a student researcher with the Key Laboratory of Electromagnetics and Radio Engineering of Beihang University, Beijing, China. In 2012, he will be a Visiting Scholar with the Department of Electrical and Computer Engineering Center for Microwave and Radio Frequency of National University of Singapore. His research interest includes extraordinary transmission phenomena, modal methods, resonances, analytical and numerical methods for guiding, resonant, periodic and nonperiodic rough surfaces, periodic structures. $\mathrm{He}$ is also interested in antennas and scattering. 


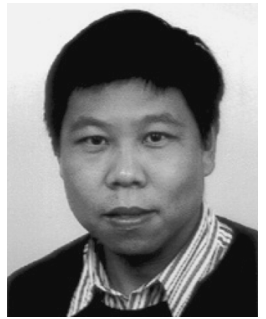

Jungang Miao was born in Hebei, China, in July 1963. He received the B.S.E.E. degree from the National University of Defence Technology, Changsha, China, in 1982, the M.S.E.E. Degree from Beihang University (BUAA), Beijing, China, in 1987, and the Dr. rer. nat. degree in physics from the University of Bremen, Germany, in 1998 .

From 1982 to 1984 , Dr. Miao was working in Beijing with the Institute of Remote Sensing Instrumentation, Chinese Aerospace, where he developed space-borne microwave remote sensing instruments. From 1984 to 1993, he worked at the Electromagnetic Laboratory of BUAA - doing research and teaching in the field of microwave remote sensing. In 1993, he changed to the Institute of Environmental Physics and Remote Sensing, University of Bremen, Germany, as a staff member doing researches on space-borne microwave radiometry. In October 2003 he returned to BUAA and since then he has been taking the Chair Professor position at the Electromagnetic Laboratory of BUAA. Dr. Miao's research areas are electromagnetic theory, microwave engineering and microwave remote sensing of the atmosphere, including sensor development, calibration and data analyses.

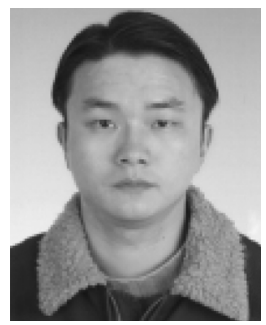

Sheng Hong (M'10) received the B.S. degree in electronic and information engineering from Haerbin Engineering University, Haerbin, China in 2003, and the Ph.D degree in communication and information system from the Beihang University, Beijing, China, in 2009 , respectively.

He had been invited as a visiting scientist in Electrical and Computer Engineering at the Georgia Institute of Technology, Atlanta, from 2007 to 2008 . He is a Graduate Student Advisor with the Beihang University at the School of Reliability and System Engineering. His research interests include Wireless Communication technology, Electromagnetics and the design and test of VLSI.

Dr. Hong is an IEICE member.

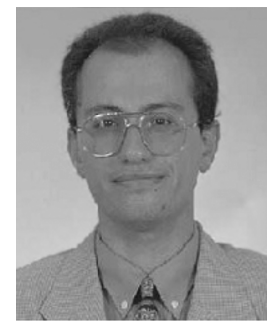

Manos M. Tentzeris (SM'03) received the Diploma degree in electrical and computer engineering from the National Technical University of Athens, Athens, Greece, in 1992, and the M.S. and Ph.D. degrees in electrical engineering and computer science from The University of Michigan at Ann Arbor, in 1993 and 1998, respectively.

He is currently a Professor with School of Electrical and Computer Engineering, Georgia Institute of Technology, Atlanta. During the summer of 2002, he was a Visiting Professor with the Technical University of Munich, Munich, Germany. He has authored or coauthored over 120 papers in refereed journals and conference proceedings and six book chapters He has helped develop academic programs in highly integrated packaging for $\mathrm{RF}$ and wireless applications, microwave MEMs, SOP-integrated antennas and adaptive numerical electromagnetics (FDTD, multiresolution algorithms). $\mathrm{He}$ is the Georgia Tech NSF-Packaging Research Center Associate Director for RF Research and the RF Alliance Leader. He is also the Leader of the Novel Integration Techniques Sub-Thrust of the Broadband Hardware Access Thrust of the Georgia Electronic Design Center (GEDC) of the State of Georgia.

Dr. Tentzeris is member of the Technical Chamber of Greece. Hewas the 1999 Technical Program co-chair of the 54th ARFTG Conference, Atlanta, GA. He is the vice-chair of the RF Technical Committee (TC16) of the IEEE Components, Packaging, and Manufacturing Technology (CPMT) Society. He was the recipient of the 2003 IEEE CPMT Outstanding Young Engineer Award, the 2002 International Conference on Microwave and Millimeter-Wave Technology Best Paper Award (Beijing, China), the 2002 Georgia Tech-Electrical and Computer Engineering (ECE) Outstanding Junior Faculty Award, the 2001 ACES Conference Best Paper Award, the 2000 NSF CAREER Award, and the 1997 Bes Paper Award, International Hybrid Microelectronics and Packaging Society. 